Aus der $\mathbf{k}, \mathbf{k}$. dermatologischen Universitätsklinik in Wien.

(Prof. Dr. Gustav Riehl.)

\title{
Ein Beitrag zur Kenntnis des Sarcoma idiopathicum multiplex haemorrhagicum (Kaposi).
}

Von

\author{
Dr. August Halle.
}

(Hiezu Taf, X.)

Im Jahre 1872 beschrieb Kaposi (1) ein Krankheitsbild, welches er "idiopathisches multiples Pigmentsarkom" nannte, im Jahre 1894 aber mit dem ihm passender erscheinenden Namen "Sarcoma idiopathicum multiplex haemorrhagicum" ${ }^{\text {" be- }}$ legte. Er sah diese Krankheit als "eine ganz eigenartige und typische Form der Sarcomatosis cutis" an, welch letztere wiederum von ihm mit der Mycosis fungoides, der Lymphodermia perniciosa und dem Sarcoma proprie dictum zu der einen Gruppe der "sarkoiden Geschwülste" zusammengefaßt wurde.

In Anbetracht der Sonderstellung, welche das Sarcoma idiopathicum multiplex haemorrhagicum namentlich in klinischer Beziehung einnimmt, und mit Rücksicht auf die gerade in neuerer Zeit unter diesem Namen sich mehrenden Publikationen von Fällen, die nur zum Teil der Kaposischen Beschreibung entsprechen, erscheint uns ein Hinweis auf das von diesem Autor entworfene klinische Bild nicht unangebracht. Seinem Lehrbuche (2) entnehmen wir folgende Schilderung:

„Dasselbe (das Sarcoma i. m. h.) beginnt stets zugleich an beiden Füßen und Händen, Planta und Vola, Hand- und 
Fußrücken und schreitet mittels diskreter Produktionen über die Unter- und Oberschenkel und Arme vor, bis es nach 2 bis 3 Jahren auch im Gesichte und auf dem Stamme erscheint. Es entstehen schrotkorn-, erbsen- bis bohnengroße, rotbraune, später blaurot werdende, rundliche, mäßig derbe Knoten, die teils diskret und unregelmäßig situiert sind, teils zu Gruppen und diffusen Infiltraten von Kreuzer- bis Flachhandgröße aneinanderreichen. Füße und Hände sind an der Streck- und Beugeseite polsterartig aufgetrieben, knollig verdickt, unförmlich, bei Druck und auch spontan sehr schmerzhaft, die Finger spindelförmig verdickt, von einander gedrängt, das Gehen und Hantieren wegen der Starrheit der Haut in hohem Grade behindert.

Die älteren Knoten sinken nach mehrmonatlichem Bestande ein unter Schilferung ihrer Epidermis und schwinden teilweise sogar gänzlich unter Hinterlassung sehr dunkler, pigmentierter, narbiger Gruben. Die aus Knotengruppen bestehenden Plaques atrophisieren ebenfalls im Zentrum und bilden so später einen die mittlere pigmentierte Narbengrube umrahmenden, gekerbten derben, braunroten, mit harten Schuppen bedeckten Wall. Manche Knoten werden auch weich, aber es kommt selten zu Ulzeration oder Massennekrose."

Weiterhin heißt es:

„Nach 2 bis 5 Jahren, wohl auch noch später, erscheinen auch Knoten von Bohnen- bis Nußgröße auf den Augenlidern, der Nase, auch an ihrer Schleimhaut, der Wange, Lippe und an verschiedenen Stellen des Stammes, die zum Teile dunkelblaurot, schwammig sich anfühlen und auch von der Oberfläche her zerfallend ein blutig suffundiertes Gewebe zu Tage legen.

Drüsenschwellung scheint von gelegentlich sympathischer abgesehen, wie z. B. bei Gangrän am Fuße, diesem Sarkomtypus nicht eigentïmlich zu sein. Um diese Zeit stellt sich Fieber, blutige Diarrhoe, Haemoptoe, Marasmus und alsbald der Tod ein.

Bei der Sektion findet man die gleichen blutreichen, fleischfarbenen Knoten in großen Mengen in der Lunge, Leber, Milz, im Herzfleisch, im Tractus intestinalis, besonders dicht gedrängt und nekrotisch zerfallen im Colon descendens." 
Diesem eng umgrenzten Krankheitsbilde entsprechen nun manche der in den letzten Jahrzehnten veröffentlichten Fälle von multipler Hautsarkomatose, viele andere dagegen müssen als andere Formen der Sarcomatosis cutis angesehen werden, deren systematische Einteilung aber bisher noch nicht endgültig gelungen ist. Schuld an dieser Unklarheit trägt einerseits die Neigung mancher Autoren mit jedem veröffentlichten Falle auch einen neuen Typus aufstellen zu wollen, andrerseits die Verschiedenartigkeit des Standpunkts, von dem aus die einzelnen Autoren die betreffenden Krankheitsbilder beurteilen zu müssen glaubten.

Während nämlich viele das klinische Bild als allein maßgebend ansahen, nahm z. B. Un n a (3) eine Einteilung rom anatomischen Standpunkte aus vor und zwar unterschied er dermale und hypodermale multiple Sarkome; von den dermalen zählt er 4 Formen auf:

1. Sarcoma multiplex cutaneum durum album.

2. Sarcoma multiplex cutaneum durum pigmentosum (Typus Piffard).

3. Sarcoma multiplex cutaneum molle (Typus Neumann).

4. Sarcoma multiplex cutaneum gummatodes (Typus F un kHy de).

Diesen 4 dermalen Sarkomtypen, welche alle das Gemeinsame haben, daß sie unregelmäßig über den ganzen Körper zerstreut sind, stellt Unna eine Gruppe systematisierter Sarkome gegenüber, welche als dunkle blau- bis braunschwarze Knoten sich zuerst an den Händen und Füßen lokalisieren. Diese von genanntem Autor als „Acrosarcoma multiplex cutaneum teleangiectodes (Hebra-Kaposi) " bezeichnete Gruppe entspricht dem Sarcoma idiopathicum multiplex haemorrhagicum, welches allein den Gegenstand unserer Abhandlung bilden soll.

Perrin (4) teilt alle Sarkome erst anatomiseh in melanotische und nicht melanotische, dann diese beiden Hauptgruppen wieder klinisch in primäre und sekundäre. $\mathrm{Zu}$ den nicht melanotischen primären Sarkomen zählt er den Typus Kaposi.

$\mathrm{Schwimmer}$ (5) unterscheidet nur das sogenannte chirurgische Sarkom und das ron Kaposi beschriebene Pigmentsarkom.

De Amicis (6) zählt 3 Arten von Sarkomatosis der Haut auf:

1. Sarcomatosis cutis non pigmentosa.

2. Sarcomatosis melanotica. 
3. Sarcomatosis multipla idiopathica pigmentata et haemorrhagica.

Pelagatti (7) teilt die Sarkome ein in die eigentlichen Sarkome und die Sarkoide oder falschen Sarkome.

Die ersteren ordnet er folgendermaßen:

Erster Typus: Primäres multiples Sarkom mit den Varietäten:

a) Akrosarkoma Kaposi,

b) das multiple maligne Sarkom.

Zweiter Typus: Das solitäre Sarkom.

Dritter Typus: Das multiple sekundäre Sarkom mit den 3 Varietäten:

a) generalisiertes von einem entzündeten kongenitalen Naevus ausgegangenes,

b) id. von einem primären Sarkom der Haut,

c) id. von einem primären nicht in der Haut lokalisiertem Sarkom ausgegangenes Sarkom.

Die Sarkoide teilt er ein in:

1. Sarkoide nach dem Typus von Boeck.

2. Sarkoide mit zahlreichen zerstreuten Knötchen.

3. Sarkoide in Gestalt von großen 'Tumoren.

4. Durch Fremdkörper verursachte Sarkoide.

Lieberthal (8) ist der Ansicht, daß das idiopathische multiple Pigmentsarkom und die Sarcomatosis cutis denselben Prozeß mit gewissen Modifikationen darstellen, da die aufgestellten klinischen und histologischen Unterscheidungsmerkmale keine durchgreifenden seien.

Diese wenigen aus der Literatur geschöpften Proben mögen genügen, um die auf diesem Gebiete der Dermatologie bestehende Uneinigkeit der Autoren zu kennzeichnen. Bei der Durchsicht der Literatur konnten wir uns des Eindrucks nicht erwehren, daß mit der Anzahl der Gruppen, in welche dieser oder jene Autor die Sarcomatosis cutis einteilte, auch gleichzeitig die Unsicherheit wuchs betreffs der Frage, in welche Gruppe der jeweilige Krankheitsfall denn nun eigentlich einzureihen sei.

Solange über die Sarcomatosis cutis die Anschauungen so geteilt sind und solange die Ätiologie und Pathogenese dieser Erkrankungen so in Dunkel gehüllt bleiben wie bisher, hat jede systematische Einteilung einen mehr oder minder problematischen Wert. Der e inzige Standpunkt, von dem aus sich schon heute eine gewisse Sonderung einzelner Gruppen 
vornehmen ließe, ist der $\mathrm{klinische;} \mathrm{aber} \mathrm{auch} \mathrm{nur} \mathrm{bis} \mathrm{zu}$ einem gewissen Grade, da es zweifellos zwischen den zahlreichen bisher aufgestellten Gruppen unzählige Übergänge gibt.

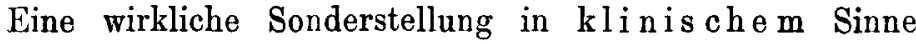
nimmt nun u. E. das Sarcoma idiopathicum multiplex haemorrhagicum ein und zwar wegen seines eminent charakteristischen Beginns und Verlaufs, den es mit keiner anderen Form der Sarcomatosis cutis gemeinsam hat. Alle jene Fälle, welche in dieser oder jener Hinsicht Ausnahmen von dieser Form darstellen sollen, gehören u. E. überhaupt nicht hierher.

Eine Einteilung der verschiedenen Sarkomformen in histologischem Sinne vorzunehmen ist bisher auch nicht geglückt, da - wie z. B. gerade beim Sarcoma i. m. h. - nach den Beschreibungen offenbar ganz identische mikroskopische Bilder bald als Sarkome, bald als Granulationsgeschwülste gedeutet werden, und die Anschauungen hierüber, wie wir im histologischen Teil unserer Abhandlung zeigen werden, noch sehr geteilt sind. Trotzdem haben aber die meisten Autoren an der Bezeichnung "Sarkoma" für den Typus Kaposi festgehalten.

Sellei (9) machte den Vorschlag, die uns beschäftigende Krankheit als "Granuloma multiplex haemorrhagicun" zu bezeichnen.

Pospelow (10) schlug die Bezeichnung „Acroangioma haemorrhagicum" vor.

Unna wählte - wie schon erwähnt - den Namen "Acrosarcoma multiplex cutaneum teleangiectodes".

Indem wir uns jetzt lediglich dem Sarcoma idiopathicum multiplex haemorrhagicum zuwenden, bringen wir zunächst die Krankengeschichten von 4 Fällen, die Herr Professor Dr. Riehl, Direktor der dermatologischen Klinik des k. k. allgemeinen Krankenhauses in Wien, uns gütigst überlie $\beta$ und von denen wir die 3 letzten selbst zu beobachten Gelegenheit hatten:

I. J. M., 69 Jahre alt, verwitwet, aus Ödenburg in Ungarn, Schuhmacher, wurde aufgenommen am 6. Juni 1901.

A namnese: Aus den schwer verständlichen Angaben des Kranken ist nur zu erfahren, daß das Leiden seit etwa einem Jahre besteht.

Status praes.: Am ganzen Körper zerstreut, insbesondere aber an den Extremitäten zahlreiche erbsen- bis wallnußgroße, violettgefärbte Tumoren, über denen die Haut unverschieblich ist. Besonders zahlreich 
erscheinen sie an den Händen und Füßen, an den Ohren und am Skrotum; einzelne davon, namentlich an den Handrücken, scheinen dem Verlauf der oberflächlichen Venen zu folgen.

An der linken großen Zehe, an deren Endglied ebenfalls ein solcher Knoten sitzt, erscheint die Haut in Form von violett gefärbten drusigen Warzen verdickt.

Diagnose: Sarcoma idiopathicum multiplex haemorrhagicum.

Ther a pie: Injektionen von Natr. arsen.

29./VI. Die verrucösen Exkreszenzen an der linken großen Zehe sind zurückgegangen, die Krustenbildung ist geringer. Viele Tumoren zeigen im Zentrum eine Delle.

7./VII. Hinter dem rechten Gaumenbogen ein an der Gaumenwand mit dünnem Stiel aufsitzender blauvioletter, haselnußgroßer Tumor, der an mebreren Stellen eitrig belegt erscheint; Tonsillen selbst frei.

10./VII. Stärkere Schmerzen in der linken großen Zehe.

Injektionen von Natr. arsen.

17./X. Zinkpflaster. Die Schmorzen bestehen fort.

28./X. Enukleation der linken großen Zehe wegen fortbestehender Schmerzen und dadurch bedingter Schlaflosigkeit.

Operation unter Schleichanästhesie.

24./XI. Operationswunde geheilt.

Patient auf Wunsch entlassen, ungeheilt.

II. B. L., 64 Jahre alt, verheiratet, Händler aus Olkusz in Rußland, wurde am 12. Mai 1903 aufgenommen.

An amnese: Patient überstand vor 8 Jahren die Cholera. Er hat 9 gesunde Kinder. 3 seiner Schwestern starben an unbekannten Krankheiten. Ein Bruder und eine Schwester sind gesund.

Beginn des Leidens vor 4-5 Jahren mit Auftreten eines dunklen schmerzlosen Flecks am rechten Handrücken; bald darauf erschien ein ähnlicher Fleck am linken Fuß. Kein Juckreiz.

Vor $1 \frac{1}{2}$ Jahren griff die Affektion vom Rücken der rechten Hand auf den Zeigefinger und allmählich auch auf die übrigen Finger über. Die linke Hand wurde vor 5-6 Monaten ergriffen, etwas später der rechte $\mathrm{FuB}$, welcher alsbald schmerzhaft wurde.

Status pra.es.: Am Dorsum der rechten Hand - und zwar über den Metakarpen des 4. und 5. Fingers - findet sich ein fünfkronenstäckgroßer Herd von unregelmäßiger Begrenzung, der sich auch auf die ersten Phalangen des 3. und 4. Fingers erstreckt und hier mit unrugelmäßigen Zacken endigt, blaurot verfärbt aussieht und bei Palpation sich als pseudoödematöses Gewebe repräsentiert, das nach Verdrängung der Hyperämie leicht pigmentiert erscheint.

Ein zweiter Herd findet sich über dem Köpfchen des Metatarsus indicis und der ersten Phalange desselben.

Beide Herde sind sehr scharf begrenzt. Innerhalb des letzteren Herdes finden sich sowohl am Rande als auch innerhalb der sonst ganz 
gleichartig aussehenden Hautveränderung kleine, reiskorngroße, leicht über das Hautniveau emporragende Knötchen.

Ein dritter Herd, isoliert stehend, findet sich am Daumen, seinen Metakarpus und die erste Phalange einnehmend; er erstreckt sich bis auf die Beugefläche des Handgelenks. Ein weiterer Herd sitzt an der Palma manus und zwar dem Metakarpus des 2. und 3. Fingers entsprechend. Der 2., 3., 4. und 5. Finger der rechten Hand weisen Gestaltsveränderungen auf, und zwar erscheint die 1. und 2. Phalange senkrecht abgebeugt und fixiert, die Fixation der 1. Phalange in der Verlängerung der Metakarpen.

Die Haut sowohl über den bezeichneten Fingern als auch über dem Dorsum manus fast bis zum Bereiche des Handgelenks ist in folgender Weíse verändert: Sie ist rotbraun, von erweiterten Gefäßen durchsetzt, atrophisch. An den Stellen, wo die Hyperämie sich zurückgebildet hat, sieht die Haut mehr graurot aus. Die Herde sind vielfach von einer $2-3 \mathrm{~mm}$ breiten, sehr stark pigmentierten, sicher aus Hämorrhagien hervorgegangenen Linie umsäumt.

An der linken Hand nimmt ein Herd den ganzen Daumen ein und zwar sowohl den Metakarpus als auch die Phalangen mit Ausnahme des Nagelgliedes, sowohl beuge- als streckwärts. Hier finden sich dieselben Erscheinungen wie an der rechten Hand. Der dorsale Herd der Finger geht auch radialwärts auf die Beugeseite über und erreicht hier ungefähr die Mitte der Hohlhand, sonst alle Finger überziehend.

Innerbalb dieses Herdes finden sich zahlreiche stecknadelkopf- bis linsengroße blauviolette Knötchen sehr dicht aneinandergedrängt, wodurch man den Eindruck gewinnt, als bestände hier eine flache papilläre Wucherung.

Kleine flache Scheibenformen finden sich sonst $4-5$ an der Zahl am Thenar. Diese zeigen im Zentrum leichte Involution und sind leicht über das Hautniveau hervorragend. Auch an der Streckseite des rechten Vorderarms finden sich spärliche, zerstreut stehende, linsengroße Herde.

Ferner finden sich: an der Beugeseite des Handgelenks in der Nähe der Cubita ein subkutan sitzender, sehr leicht beweglicher, nicht derber Knoten; an der linken Hand eine ganze Reihe solcher Knoten, die schief über den radialen Rand nach der Cubitalgegend hinüberziehen; einzelne auch am ulnaren Raude des Vorderarms Die Drüsen in der Axilla sind nicht geschwollen, dagegen die Drüsen in der Cubitalgegend bis zur Größe einer welschen Nuß, Ähnliche Tumoren finden sich am Oberarm.

Beide Füße sind fast symmetriseh in gleicher Weise ergriffen, und zwar findet man beiderseits einen Herd, der ungefähr von deu Köpfchen der Metatarsi bis zu der ersten Phalange der Zehen reicht, die große Zehe dorsalwärts ganz überzieht, um nach rückwärts in einer Linie zu endigen, die gedacht werden kann vom Malleolus internus zur Basis des Köpfchena der 3. Phalange, von hier über den inneren Fuforand auf die Planta übergeht und über den äußeren Fußrand auf das Dorsum zurück- 
kehrt. Auch über dieser ganzen Partie ist die Haut blaurot, an einzelnen Stellen schwarz verfärbt.

Auf der Planta pedis finden sich innerhalb eines Herdes, der leicht ödematös, auf Druck schmerzhaft ist, scharf begrenzte, linsengroße, blaurote Knötchen.

Einzelstehende Herde finden sich anch sonst noch in der Gegend der Malleolen, linkerseits und rechterseits auch über dem untersten Drittel der Tibiakante. Alle Herde sind ödematös; beim öfteren Darüberstreichen läßt sich das Ödem verdrängen und es bleibt eine graue Verfärưung zurück. Einzelne Herde baben einen leicht gelblich gefärbten Saum, der aber bei den meisten Herden verloren gegangen ist. Oft sind die Herde scheinbar serpiginös angeordnet, immer aber mit unregelmäßigem Kontur begrenzt. Die Herde springen ungefähr $2 \mathrm{~mm}$ über das Hautnivean empor.

Ähnlich wie am Oberarm sind auch am Unterschenkel innerhalb der Cutis unregelmäßigere und diffusere Knotenformen, an der Streckseite beider Oberschenkel im oberen Drittel ganz symmetrische Tumoren nachzuweisen.

Diag nose: Sarcoma idiopathicum multiplex haemorrhagicum.

Therapie: Injektionen von Natr. arsenicos.

20./VII. Blutbefund:

$$
\begin{array}{lr}
\text { Hämoglobin (Fleischl) } & 51 \% \\
\text { Erythrocyten . . . . . } 2706000 \\
\text { Leukocyten . . . . . . } 13000
\end{array}
$$

Die roten Blutkörperchen verhalten sich normal; die polynucleären Leukocyten überwiegen.

24/VII. Am rechten Sprunggelenk zahlreiche rote Knötchen von Stecknadelkopf- bis Linsengröße, ziemlich derb, unter dem Fingerdruck abblassend, leicht schuppend. Excision eines Knotens an der Fußsohle.

Am 31. August wird der Patient auf seinen Wunsch entlassen, nachdem während des Spitalaufenthaltes vereinzelte Tumoren sich zurückgebildet, an anderen Stellen aber doch wieder neue aufgetaucht sind. in Rußland.

III. M. M., 58 Jahre alt, verheiratet, Goldschmied aus Czernichew

A namnese: Der Patient gibt an, $d a \beta$ er vor 2 Jahren eine von Juckreiz begleitete Verdickung der Haut wahrnahm. Der Zustand verschlimmerte sich allmählich.

Status praes.: Beide Füße sind ziemlich symmetrisch erkrankt, der linke mehr; dortselbst sind die ganze Sohle, die Zehen in ihrer Totalität und die innere Seite des Fußes blaurot verfärbt und die Haut hebt sich in wulstigen Verdickungen über das normale Hautniveau empor.

Die Zehen erscheinen plump und ihre Haut ist von feinhöckeriger Beschaffenheit. An der Planta sind die Unebenheiten so stark, daß die Haut papillomatös aussieht. Diese Partien sind ziemlich schmerzhaft. 
Auch an den Unterschenkeln ist die Haut leicht verdickt, und es finden sich dort isoliert stehende, erbsen- bis guldengroße, blaurote, ziemlich flache Knoten, welche meistens zentral eingesunken sind und von welchen in die Umgebung gelbliche Verfärbungen ausstrahlen. Die Tumoren nehmen zentripetalwärts an Zahl ab, doch finden sich noch vereinzelte in der Kniekehle und an der Rückseite der Oberschenkel. Auch $z$ wischen diesen Knoten fühlt sich die Haut höckerig an, obwohl sie normal aussieht.

Am rechten Fuße sind die Erscheinungen weniger ausgeprägt.

Die Interdigitalfurchen und die seitlichen Teile der Phalangen sind blaurot infiltriert.

Die Glans penis und der Sulcus coronarius weisen mehrere flache blaurote Infiltrate auf, welche sich kartenblattartig aufheben lassen und sich knorpelhart anfühlen.

An der rechten Brustseite ein Herpes zoster mit bereits vertrocknenden Bläschen.

Diagnose: Sarcoma idiopathicum multiplex haernorrhagicum.

Therapie: Arsen; äußerlich essigsaure Tonerde.

20./VI. Solut, arsen. Fowl.

30./VI. Röntgenbestrablung, Umschläge mit essigsaurer Tonerde.

15./VII. Leichte Reaktion auf die Röntgenbestrahlung vom 30./VI. Schmerzempfindung in den beiden Fußgelenken. gegeben.

25./VII. Borsalbenverbände; inverlich wird weiter Sol, arsen. Fowl.

20./VIII. Schmerzen im linken Fuße. An der rechten Tonsille circumscripte bläulichrote Verfärbung.

23/X. Injektion von Natr, arsenicos. Eisumschläge auf die Füße

30./X. Jeden zweiten Tay Injektionen von Natr. arsenicos.

Blutbefund :

$$
\begin{aligned}
& \text { Hämaglobin (Fleischl) } \quad 55 \% \\
& \text { Erythrocyten . . . . . . . . } 989000 \\
& \text { Leukocyten . . } 9600
\end{aligned}
$$

Die roten Blutkörperchen sind normal, die polynucleären weißen überwiegen.

3./XI. Excision zweier bohnengroßer Knoten der rechten Fußsohle.

24./XI. Am (s. Zt. bestrahllen) rechten Fuße ist eine leichte Abschwellung und Abblassung zu bemerken. Patient hat beim Auftroten weniger Schmerzen.

21./XII. Exulzeration der an der linken Fußsohle befindlichen Sarkomknoten. Borsalbe.

Wegen penetranten Geruchs der Geschwüre Jodoformkohlenpulver.

15.I. Das Geschwür an der linken Fußsohle fast handtellergroB, der Grund uneben mit mißfarbigem Eiter belegt. Der Rand ist unregelmäßig und stark eleviert.

30./I. Der Geschwürsrand beginnt sich gbzuflachen und die Sekretion ist im Abnehmen begriffen. 
5./II. Das Epithel schiebt sich an einzelnen Stellen vom Rande gegen das Zentrum des Geschwürs vor.

9./II. Vor Ablauf des Heilungsprozesses wird Patient auf eigenes Verlangen entlassen.

In jenen Hautbezirken, die während des Spitalaufenthaltes der Röntgenbestrahlung ausgesetzt werden konnten, sind neue Tumoren nicht aufgetreten; die schon vorhandenen zeigen vielfach Spuren von Rückbildung.

Die Bestrahlung wurde in der Weise vorgenommen, daß an einzelnen Tagen ein abgegrenzter Hautbezirk nur einmal 10-15 Min. hindurch bestrahlt wurde. Die ziemlich harte Röhre befand sich in einem durchschnittlichen Abstande von $15 \mathrm{~cm}$ von der Haut. Die angewandte Stromstärke betrug 3 Milli-Ampère. Die Reaktion trat in Form einer leichten Rötung durchschnittlich nach 6-8 Tagen auf.

IV. J. K., 65 Jahre alt, Agent aus Wien, wurde am 31. Juli 1903 aufgenommen.

Anamnese: Die Krankheit begann vor 4 Jahrea am unteren Drittel des Unterschenkels als eine Verfärbung, die der Patient auch bei ihrem Fortschreiten nicht beachtete, da sie ihm keine Schmerzen verursachte. Trotzdem auch bald die Fubsohlen befallen wurden, hatte er zunächst keine Schmerzen. Erst in letzter Zeit stärkere Beschwerden beim Gehen.

Status praes.: Beide Füße sind symmetrisch ergriffen, vor allem die Sohlen und Fußrücken; nur die Ränder sind frei.

Die Haut an diesen Stellen ist blaurot verfärbt und stark verdickt, leicht schuppend. tiefblau.

Die Färbung ist fleckig, hell und dunkel, an manchen Stellen

Die Zehen sind plump verdickt, tiefblan verfärbt; nur die beiden kleinen Zehen sind an der Außenseite frei.

Die Unterschenkel weisen bis zur Mitte ebenfalls eine blaurote verdickte Haut auf; überall sind etwa erbsengroße, ziemlich weich sich anfühlende, dunkelbraunrote Knötchen eingelagert. - An den Oberschenkeln und am Bauch finden sich zahlreiche, unregelmäßig geformte, guldengroße und noch größere, blaurote Infiltrate in die Haut eingelagert.

Die Rückseite der Oberschenkel ist mit größeren, aus einzelnen kleinen Flecken konfluierenden, plattenförmigen Infiltraten von Handflächengröße besetzt - Ad nates fühlt sich die Haut höckerig an, und diese Stellen sind teils blaurot, teils normal gefärbt.

Der Handrücken weist eine verdickte, braunrote Haut auf.

Die Haut zwischen den Fingern füblt sich polsterartig an.

Am Oberarm finden sich unregelmäßig geformte, fleckige, blaßrote, plattenförmige Intiltrate in die Haut eingelagert, die sich ebenfalls ziemlich weich anfühlen. - In inguine beiderseits etwa bohnengroße Lymphdrüsen. 
Am Penis mehrere blaurote Infiltrate. - Die Haut des rechten Nasenflügels und der Ohrmuschelränder ist leicht verdickt und braunrot verfärbt.

Diagnose: Sarcoma idiopathicum multiplex haemorrhagicum.

Therapie: Pil. asiaticae, Borsäure-Umschläge, Radiotherapie.

5./IX. An der hinteren Rachenwand rechts findet sich ein längsovales, scharf begrenztes, blaurotes Infiltrat.

24./X. Gebessert entlassen.

22./I. 04. Der Patient wird ungefähr in demselben Zustande wieder aufgenommen, in dem er sich zur Zeit seiner Entlassung im Oktober befand. Nur sind die Infiltrate am rechten Fuße weniger derb und ihre Farbe ist deutlicher ins braune übergegangen. Die Hände sind frei.

Blutbefund :

Haemoglobin (Fleischl) $\quad .52 \%$
Erythrocyten . . . . 2930000
Leukocyten . . . . 6600
Neutrophile . . . . . $72 \%$
Lymphocyten . . . . $21 \%$
Große mononukl. . . . . $44^{4} \%$
Eosinophile . . . . . $1 / 2 \%$

23./I. An der polsterartig verdickten linken Fußsohle werden zwei etwa kreuzergroße Infiltrate excidiert. Es erfolgt starke venöse Blutung (ca. $1 / 3 l$ Blut). Der Fuß schwillt ab. Der Kranke fühlt sich subjektiv erleichtert.

Der Patient wird an sämtlichen sarkomatösen Herden bestrahlt und bekommt innerlich Levico.

10./III. Der Patient wird gebessert entlassen. Viele Infiltrate bilden sich zu Pigmentationen zurück, andere bestehen unverändert fort.

Der rechte $F u ß$ ist abgeschwollen und gebrauchsfähig, der linke polsterartig geschwollen, gerötet und schmerzhaft.

18./IV. Wiederaufnahme.

In der Zeit vom 21./IV. bis 4./VI. wird der Patient 18mal je 15 Min. lang bestrahlt. Verbände mit essigsaurer Tonerde und Borsalbe. Innerlich : Solut. Fowl. steigend bis 10 Tropten pro die.

Nach Bestrahlung sämtlicher Effloreszenzen trat Reaktion ersten Grades auf mit gleichzeitiger Abnahme der Infiltration und Pigmentation.

An den diffus ausgebreiteten Infiltrationen der Innenfläche beider Oberarme bemerkt man eine deutliche Depigmentation, welche zentrifugal erfolgt, so daß dieselben von einem dunkelbraunroten Rande der ursprünglichen Verfärbung - umsäumt erscheinen.

$18 /$ VI. Gebessert entlassen.

Mit vergleichenden Rückblicken auf unsere Krankengeschichten wollen wir nun in folgendem eine Übersicht über die Anschauungen der Autoren bringen, wie sie in den wichtigsten Publikationen der innerhalb 30 Jahren riesig angewachsenen Literatur zur Geltung kommen: 
Bezüglich der Ätiologie ist man seit $\mathrm{Kaposi}$ in der Erkenntnis um keinen Schritt vorwärts gekommen.

Schon Perrin nahm an, daß Mikroorganismen eine Rolle spielen.

Pringle (11) fand in zwei Fällen Bazillen, über deren Wesen er sich aber eines Urteils enthielt.

Philippson (12) glaubt, daß irgend ein Virus von außen in die Haut gelange und an der Eintrittsstelle die $\mathrm{Wu}$ cherung von Spindelzellen oder die Bildung von cavernösen Haem- bzw. Lymphangiomen veranlasse. Eine Zeitlang hier lokalisiert komme das Virus alsdann in den Blutkreislauf und auf embolischen Wege neuerdings in die Haut, u. zw. zunächst in die Hand- und Fußregionen. Von hier aus rückt es durch die Lymphwege zentripetal weiter vor.

In jüngster Zeit mehren sich nun jene Stimmen, welche sich für die infektiöse Natur des Leidens aussprechen, doch sind alle nach dieser Richtung hin angestellten Versuche bisher erfolglos gehlieben. (Frosch, Reale, Bernhardt, Semenow u. a.)

Semenow (13) siebt auf Grtnl zweier zur Sektion gekommener Fälle, bei denen anamnestisch sowie bei objektiver Untersuchung Störungen von Seiten des Nervensystems zu konstatieren waren, die auch mikroskopisch sich bestätigten, das Sarcoma pigmentoides idiop. $\mathrm{m}$. cutis (K a p o si) als ein nervöses Leiden an, welches höchst wahrscheinlich durch paralytische Angioveurose hervorgerufen sei.

Campana (14) fand in einem seiner Fälle Sarkom der Nerven und der Haut, in einem anderen Sarkom der Haut und ein Neurofibrom. Beim ersten Falle fanden sich trophische Veränderungen der Nägel, der Haut und des Unterhautzellgewebes, Stauungen in den Blut- und Lymphgefäßen, Sensibilitäts- und auch Motilitätsstörungen. Die sarkomatösen Tumoren standen manchmal im Zusammenhange mit dem Nervenverlauf. Daraus zog Campana den Schluß, daß die Sarkomatosis eine neurotische Affektion sei.

Diese Anschauung teilt auch Tinzew auf Grund eigener Beobachtung.

Ob die von letztgenanntem Autor und auch von anderen (z. B. Semenow, Schwimmer) erwähnte, dem Beginn des Leidens vorausgegangene Durchnässung resp. Erkältung der Füßje mit dem Leiden in irgend eiven kausalen Zusammenhang gebracht werden kann, muß dahingestellt bleiben. Erwähnen möchten auch wir die Angabe eines unserer Patienten (IV), daß nach wiederholtem Gebrauch von einem Arzte verordneter kalter Bäder sein Zustand sich erheblich verschlimmerte. 
Paltauf (16) hält es für wahrscheinlich, daß noch andere Ursachen als die bekannten und immer wieder in Rechnung gezogenen formativen und vitalen Reize derartige Gewebsproduktionen auslösen können, und rechnet die Sarcomatosis cutis zu den "Vegetationsstörungen".

Das auffällig häufige Vorkommen der Krankheit bei Juden legt die Frage nahe, ob nicht etwa auch eine in der Rasse liegende Disposition dem Auftreten der Krankheit günstig sei.

Vererbung und Syphilis ließen sich nie mit der Krankheit in Verbindung bringen.

Über den eigentlichen Verlauf des Leidens wäre der klassischen Schilderung $K$ aposis kaum etwas hinzuzufiugen, zumal auch alle späteren Autoren damit im wesentlichen übereinstimmen.

Charakteristisch ist ror allem der Begiun des Leidens an den Händen und Füßen. Meist findet sich anfangs eine mehr diffuse Infiltration oder ein Ödem der Extremitäten; Knotenbildung erfolgt erst später.

Bei einem Fal'e Köbners (17) zeigte sich zuerst ein juckender Fleck am Knöchel.

In 6 von den 10 Fällen Semenows (18) begann das Leiden am linken Fuß und ergriff dann kreuzweise nacheinander die übrigen Extremitäten. Dasselbe beobachteten auch wir an zweien unserer Patienten (II u. IV), während bei den beiden anderen der Sitz der ersten Affektionen anamnestisch nicht zu ermitteln war. Entsprechend diesem anscheinend sehr häufigen Beginn des Leidens am linken FuB und an der rechten Hand fanden wir auch im weiteren Verlauf die Symptome an den genannten Extremitäten stets stärker ausgeprägt als an anderen Körperstellen. Beispiele für diese Beobachtung liefern außer den schon erwähnten auch unsere Fälle I u. III.

Interessant war in einem Falle Selle is (19) und bei einem unserer Patienten die Lokalisation der Knötchen in der Richtung der größeren Venen, hauptsächlich der Vena saphena.

In einem unserer Fälle war bei einer Anzahl fast gleichzeitig aufgetretener Knötchen eine Gruppierung nach der Spaltrichtung der Haut deutlich erkennbar.

Subjektive Beschwerden fehlen anfangs meist oder sie sind gering und steigern sich erst später besonders durch die vorzugsweise Lokalisation der Knoten an der Fußsohle. Auch der Gebrauch der Hände ist oft schon frühzeitig durch die polsterähnliche Beschaffenheit der stark gespannten Haut sehr erschwert. 
Sehr auffällig ist die anfangs teigige Konsistenz der zunächst mehr hellrot gefärbten Knoten, die dem tastenden Finger vielfach das Gefühl eines ausdrückbaren und sich dann wieder füllenden Schwammes darbieten. Mit zunehmender Derbheit der Knoten wird auch ihre Farbe immer dunkler, bis sie endlich als dunkelblaue fast knorpelharte Tumoren den höchsten Grad ihrer Entwicklung erreichen.

In diesem Stadium können sie monatelang ohne makroskopisch wahrnehmbare Veränderung verharren, bis sie endlich durch Resorption mit Hinterlassung dunkel pigmentierter, mehr oder minder atrophischer Flecke verschwinden während an anderen Körperstellen neue erscheinen und denselben Entwicklungsgang durchmachen.

Dieses spontane Verschwinden der Knoten stellt u. E. eine der auffallendsten klinischen Erscheinungen des Leidens dar und wird von Kaposi (20) in folgender Weise erklärt: Er glaubt, da $\beta$ das harte Infiltrat einzelver frischer Knoten eine Art Fihrininfiltration darstelle, die durch die zahlreichen $\mathrm{Hä-}$ morrhagien veranlaßit wird. Mit der Schrumpfung und Resorption dieser Infiltrate erfolge auch gleichzeitig die Atrophie jener kleinzelligen Sarkomknoten, die in ibrem Bereiche liegen.

Schwimmer (21) dagegen ist der Ansicht, daß die in dem Gewebe auftretenden häufigen Blutungen innerhalb der Faserbündel das Sarkomgewebe auseinanderschieben, wodurch die einzelnen Sarkomzellen in die Umgebung verdrängt werden.

Sellei glaubt, daß teils die ausgetretenen Blutzellen, teils die neugebildeten Blutgefäße die Sarkomelemente vernichten.

Philippson erklärt die spontane Rückbildung durch die von ihm beobachtete mucinöse Degeneration der Zellen und durch die fibröse Veränderung der Neubildungen.

B erahardt (22) unterscheidet zwei Rückbildungstypen : a) durch Atrophie, b) durch Degeneration.

Im ersten Falle bemerkt man, daß das Knötchen nach Erlangen einer gewissen Größe sich allmählich zu verkleinern anfängt. Hauptsächlich wird es flacher, wobei in seiner Mitte cine seichte Vertiefung sichtbar wird. Die Epidermis schuppt ab. Eine solche Atrophie schreitet gewöhnlich nur bis $\mathrm{zu}$ einem gewissen Grade, auf welchem sie stehen bleibt. In einzelnen Fällen findet jedoch eine totale Atrophie statt und auf der Stelle des Sarkomknötchens bleibt dann eine stark pigmentierte Narbe zurück.

Der zweite Rückbildungstypus besteht in einer allmählichen Erweichung des Sarkomgewebes so, daß endlich ein weiches, schmutzigbläuliches, halblkugelförmiges Knötchen entsteht, in dem man die Anwesenheit einer zähen Flüssigkeit nachweisen 
kanu. Das Verschwinden des Knötchens geschieht endlich infolge Resorption der degenerierten erweichten Masse.

Bernhardt beobachtete aber auch eine große Regenerationsfähigkeit der Knoten an atrophischen, pigmentierten Stellen, die dem früheren Sitz inzwischen involvierter Knoten entsprachen, ja sogar innerhalb der nach gründlicher Excision zurückgebliebenen Narben eine auffallende Erscheinung, die sehr an das Verhalten anderer Tumoren erinnert, die wir aber sonst nirgends in der Literatur erwähnt fanden und auch an unseren Fällen nie koustatieren komnten.

Ein spontanes Ulzerieren der Knoten scheint höchst selten vorzukommen; von uns wurde es in einem Falle (III) beobachtet. Wo wir aber sonst geringfügige Ulzerationen fanden, waren sie fast stets durch die Lokalisation resp. durch traumatische Einwirkungen (FuBsohle) zu erklären. Gerade die oben erwähnte, spontane, ohne jeglichen Zerfall erfolgende Involution der Knoten gilt vielen neueren Autoren als ein Beweis dafuir, daf es sich bei dieser Krankheit nicht um echte Sarkome handeln könne, da bisher in der Pathologie mit dem Begriff des Sarkoms stets das grenzenlose Wachstum und der schließ. Jiche Zerfall verbunden war. Nun gibt es aber sicherlich auch andere echte Tumoren, wie z. B. manche Epitheliome des Gesichts, welche nur geringe Neigung zum Zerfall zeigen und ohne Ulzeration durch Bildung narbig atrophischen Gewebes teilweise verschwinden können; ebenso ist es von den Lymphosarkomen bekannt, daß sie sich hochgradig involvieren können. Mit diesem Einwurf soll aber keineswegs geleugnet werden, daß die Tumoren unseres Krankheitsbildes mit ibrer anfänglich schwammartigen Beschaffenheit, später derben Konsistenz und ihrem spontanen Verschwinden eine Besonderheit darstellen. Ob sie aber wegen dieser Sonderstellung aus der großen Gruppe der echten Geschwülste (Sarkome) auszuscheiden sind, ist eine Frage, welcher wir im histologischen Teil nähertreten wollen.

Mit Hinweis auf $\mathrm{Kaposis}$ Beschreibung möchten wir ferner das allmähliche Fortschreiten der Krankheit von den Extremitäten aus in zentripetaler Richtung hervorheben. Mit der Ausbreitung der Affeltion über den Stamm nimmt die Anzahl der Knoten häufig in ganz überraschendem Maße zu. In einem Falle Kalinderos (23) betrug die Anzahl der im Verlauf von 4 Monaten aufgetretenen Tumoren 450.

$\mathrm{Zu}$ welcher Zeit die rielfach beobachteten Tumoren der Schleimhaut auftreten, ist aus der uns zur Verfügung stehenden Literatur nicht genau zu ersehen. Bei einem unserer Fälle beobachteten wir nach 4jäbrigem Bestehen der Krank- 
heit einen etwa erbsengroßen flachen Tumor an der hinteren Pharynxwand.

Bei einem Falle Kaposis war an der hinteren Wand des Pharynx, ferner an der unteren Fläche der rechten Epiglottishälfte die Schleimhaut und Submucosa durch einen gefäßreichen, mäßig derben Knoten ersetzt.

Sedziak (24) fand in einem Krankheitsfalle, den Elsenberg als zum Typus Kaposi gehörig ansieht, den Isthmus faucium durch eine hühnereigroße Geschwulst von glatter Oberfläche und dunkelblauer Farbe ausgefüllt.

Auch über den Zeitpunkt des Auftretens der Tumoren in inneren Organen sind sichere Anhaltspunkte nicht zu gewinnen, doch darf man u. E. aus dem oft jahrelang guten Allgemeinbefinden der Kranken schließen, daß erst zuletzt der Prozeß auf innere lebenswichtige Organe übergreift. Auf diesen Punkt werden wir bei Besprechung der Sektionsbefunde noch näher eingehen.

Die Erkrankung der Knochen demonstrierte Scholtz (25) in viro bei einem von Bernhardt publizierten Falle vermittelst der Röntgenstrahlen. Bei der Durchleuchtung der Fübe fiel besonders rechts eine hochgradige Durchlässigkeit der Knochen für die X-Strahlen auf. Dieselbe war an den Phalangen, dem Metakarpus und den distalen Fußwurzelknochen des rechten Fußes so stark, daß von diesen Knochen ein deutlicher Schatten iiberhaupt kaum mehr geworfen wurde. Nach der zur Linderung der Beschwerden vorgenommenen Amputation des Fußes wurde die auf Grund jenes Befundes vermutete sarkomatöse Erkrankung der Knochen durchaus bestätigt: Die Phalangen, die Metakarpal- und distalen Karpusknochen waren teils vollständig sarkomatös zerstört und in braunrote, schwammige Massen verwandelt, teils war nur noch eine diinne Knochenlamelle erhalten, und auch in den proximalen Fußwurzelknochen und selbst im distalen Ende der Tibia und Fibula fanden sich kleinere Sarkomherde.

Auch in einem unserer Fälle (I) ergab sich nach der wegen großer Schmerzhaftigkeit vorgenommenen Enukleation der linken großen Zehe, daß die Knochen vollkommen zerstört und durch sarkomatöses Gewebe ersetzt waren.

Über den Blutbefund bei Sarcoma id. m. h. finden wir in der Literatur schwankende Angaben. In den meisten Fällen bietet die Blutuntersuchung keine Besonderheiten dar, wie z. B. auch bei unseren Patienten; zuweilen zeigen sich Abweichungen von der Norm 7. B. im Falle Geza Die ballas (26), der eine deutliche Lymphocytose aufwies. Vielleicht war dieser Befund aber ebenso zufällig, wie die auch sonst bei Sarcomatosis cutis z. B. von Joseph, Touton 
und $\mathrm{Fröhlich} \mathrm{beobachtete} \mathrm{Leukämie} \mathrm{resp.} \mathrm{Pseudoleukämie.}$ $\mathrm{Da}$, wie gesagt, ein abnormer Blutbefund nicht die Regel bildet, liegt kein Grund vor, ihn zum Symptomenkomplex zu rechnen.

Ebenso gehören Lymphdrüsenschwellungen zu den Ausnahmen und wo sie vorkamen, waren sie vielfach auch nur regionär. Tanturri (27) beobachtete sie allerdings in 6 von 8 Fällen; auch Sellei (28) betont ihr häufigeres Vorkommen.

Nachdem wir somit noch einmal die Hauptsymptome, wie sie von zahlreichen Beobachtern dargestellt werden, gestreift haben, wollen wir von zufälligen Nebenbefunden noch einen Fall von idiopathischer Hautsarkomatose verbunden mit visceraler Carcinomatose erwähnen, den Philippson (29) beschrieb.

Für eine eigentümliche Form der sogen. Sarcomatosis cutis, die er "Sarcoma idiopathicum multiplex en plaques pigmentosum et lymphangiectodes" nennt, nimmt $B$ e rn hardt (30) eiue Sonderstellung (Untertypus) in Anspruch, weil trotz einer Krankheitsdauer von 6 Jahren nur eine Extremität befallen war und keine Knoten aufwies. Die sarkomatöse Degeneration der Haut äußerte sich vielmehr in Gestalt von teigig-weichen, scharf-begrenzten Herden, welche sich über das Niveau der Haut nicht emporhoben und eine kolossale Erweiterung des Lymphgefäßsystems aufwiesen. Aber schon Kaposi war die vielfach ungewöhnliche Erweiterung der Lymphspalten bekannt; ebenso fand u. a. Philippson Neubildung von Lymphkapillaren und Lymphangiomen. Auch Sedziak (31) beschrieb eiwen "seltenen Fall von Sarcomata multiplicia cutis et lymphosarcoma tonsillae dextrae", den Elsenberg (als Referent) für idiopathisches multiples Pigmentsarkom hielt.

Als häufige Komplikation, die aber nicht zum Symptomenkomplex gehört, sei noch das Erysipel erwähnt, dessen Vorkommen bei Sarcoma id. m. h. schon von Kaposi (32) hervorgehoben wurde. Von späteren Beobachtern hat vor allem Bernhardt auf diese Tatsache hingewiesen. In einem seiner Fälle begann die Krankheit mit einem Erysipel, und er glaubt, daß letzteres durch Alteration des Lymphgefäßsystems einen güı.stigen Boden für die Entwicklung des Sarkoms geschaffen und außerdem das Eindringen eines noch unbekannten Krankheitserregers gefördert habe. In einem anderen jener von ihm beobachteten Fälle, die von rezidivierendem Erysipel begleitet wareu, zeigte es sich, daß bald nach einem Erysipel auf derselben Stelle eine große Anzahl frischer Sarkomknötchen ent- 
stand, während die schon bestehenden rasch zu wachsen anfingen. $\mathrm{Ob}$ dieses nur eine Koinzidenz sei oder auf einem kausalen Zusammenhang beruhe, läßt Bernhardt dahingestellt, doch macht er darauf aufmerksam, daß die erwähnten auffallenden Erscheinungen nur beim Sarcoma i. m. h. vorkommen, während sonst bei vielen anderen Fällen ron Sarcomatosis cutis die Tumoren unter dem Einfluß eines Erysipels gänzlich rerschwinden können.

Wir selbst möchten betreffs der Beziehungen zwischen Tumoren und Erysipel auf die Publikationen von S ta $\mathrm{y}$ z i a le (33), Lange nbuch (34) und Coley (35) hinweisen.

Ein besonderes Interesse nehmen noch die Sektionsb e funde in Anspruch, da das Befallenwerden innerer Organe den schließlich stets ungünstigen Ausgang des Leidens erklärlich macht.

Kaposi hat das Wesen der Krankheit auch nach dieser Richtung hin gekannt und über das Vorkommen der Knoten in Lunge, Leber, Milz, Herz und Darm in seiner ersten Beschreibung berichtet.

Schwimmer fand bei einem seiner Fälle hauptsächlich Tumoren im Magen.

Semenow und Campana fanden, wie schon oben erwähnt, auch sarkomatöse Veränderungen an den Nerven.

Überhaupt scheint jedes Organ befallen werden zu können, doch wurden am häufigsten Tumoren im Verdauungstraktus gefunden und hier wiederum am zahlreichsten im Kolon, wodurch die blutigen Diarrhöen und der häufig rapide Verfall der Kranken ihre Erklärung finden.

Über den Zeitpunkt des Auftretens der Knoten in inneren Organen lassen sich keine positiven Anbaltspunkte gewinnen, nur vermutungsweise kann man mit Berücksichtigung der Tatsache, daß der Verdauungskanal vorzugsweise und von allen inneren Organen am frübesten befallen wird, annehmen, daß diese überhaupt erst relativ spät ergriffen werden.

Das Allgemeinbefinden der Kranken müßte sonst doch viel trüher alteriert werden als dies nach den übereinstimmenden Angaben der Autoren der Fall ist. Überhaupt rapid verlaufende Fälle ändern an dieser Überlegung nichts.

In einem Falle Köbners fand sich bei der Sektion in den inneren Organen keine Spur von Sarkom.

Zur Ergänzung des klinischen Bildes haben wir noch hinzuzufügen, daß von der Krankheit vorzugsweise Männer befallen werden.

Unter 38 von Bernhardt zusammengestellten Fällen befanden sich nur 2 Frauen, was einem Verhältnis von $5 \cdot 25 \%$ entspricht. 
Von 50 Fällen de Amicis, betraf keiner eine Frau.

Was das Le be n s alter der Kranken anbetrifft, so scheint die früher herrschende Ansicht, daß nur das höhere Alter betroffen werde, keine allgemeine Giltigkeit mehr zu haben.

Die meisten Kranken hatten zwar das 45. Lebensjahr überschritten, jedoch berichtet $z$. B. de $\mathrm{Ami}$ is von einem

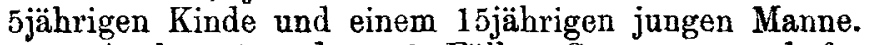

Auch unter den 10 Fällen Semenows befanden sich Kranke von 12, 22, 24 und 26 Jahren.

Betreffs der Krankheitsdauer hat Kaposi selbst schon seine ursprüngliche Ansicht modifiziert. Im Jahre 1872 gab er an, daß die Krankheit innerhalb 2-3 Jahren zum Tode führe, doch rechnete er im Jahre 1886 schon mit einer Krankheitsdauer von 10-15 Jahren.

Ein Patient Jacksons (36) erfreute sich nach 21jähriger Krankheit noch eines relativ guten Allgemeinbefindens.

Auch Elliot (37) will Fälle von so langer Dauer schon gesehen haben.

Die Diagnose des Leidens ist nicht schwierig. Von anderen Formen der Sarcomatosis cutis unterscheidet sich das Sarcoma id. m. h. durch den charakteristischen Beginn an den Extremitäten sowie durch die Eigentümlichkeit der Tumoren sich involvieren zu können.

Zuweilen können letztere Ähnlichkeit mit luetischen Produkten haben, wie in einem Falle von $\mathrm{Havas}$ (38).

D'Antona (39) ist der Ansicht, daß viele Fälle von sog. Pigmentsarkom luetischer Natur seien.

Einige Ähnlichkeit können unter Umständen die Knoten auch mit tuberösen Formen der Lepra haben.

Ferner kommen differentialdiaguostisch noch Fibrome, Neurome, Myome, der Cysticercus cellulosae und die Mykosis fungoides in Betracht, doch ist eine Verwechslung mit diesen Affektionen, wenn auch nicht immer im Beginn, so doch im weiteren Verlauf durch die Beobachtung sicher zu vermeiden.

Die Prognose ist als ungünstig zu bezeichnen. Zwar können, wie bereits erwähnt, die Kranken sich oft viele Jahre lang eines leidlichen Wohlbefindens erfreuen, doch droht schließlich stets der letale Ausgang durch die Erkrankung innerer Organe.

Anscheinende Besserungen werden häufig durch die Rückbildungsfähigkeit der Tumoren vorgetäuscht, durch welche auch 
bisher eine kritische Würdigung der vorgeschlagenen therapeutischen Maßnahmen sehr erschwert wurde.

Sequeir und Bulloch (40) glauben, daß die Krankheit in einer gutartigen und in einer bösartigen Form auftrete.

De Amicis berichtet von einem spontan geheilten Fall.

Therapie: Nach Köbners (41) Vorschlage, welcher im Jahre 1882 als erster das Arsen gegen Sarkome der Haut mit Erfolg anwandte, haben seither eine große Anzahl ron Autoren dieses Mittel auch beim Typus Kaposi versucht.

Das Arsen wurde bald in Form subkutaner Injektionen, bald per os den Kranken einverleibt.

Über die damit erzielten Erfolge lauten die Berichte nun sehr verschieden. Günstige Wirkung von der Arsenbehandlung sahen Havas (42), Vigano (43), Metscherski (44), Trapesnikow (45), Pospelow (46), Kracht (47) u. a.

De A micis stellte einen mit Arsen behandelten 15jährigen jungen Mann als geheilt vor.

Auch Sherwell (48) beobachtete eine Heilung durch Arsen.

Bei einem Patienten Jacksons verschwanden nach 4monatigem Arsengebrauch mehrere Läsionen.

Im Gegensatz zu diesen günstigen Berichten sahen Kaposi, Schwimmer, Semenow u. a. nur mäßigen resp. gar keinen Erfolg von der Arsenbehandlung.

Inwieweit rorübergehende Besserungen bei unseren $\mathrm{Pa}$ tienten auf Rechnung des Arsens zu setzen sind, entzieht sich unserer Beurteilung.

Von anderen therapeutischen Vorschlägen sei der d'Antonas erwähnt, welcher das Quecksilber auch in solchen Fällen für günstig wirkend erklärt, die nicht mit Syphilis in Verbindung gebracht werden können.

Uiorth (49) behandelte die Tumoren ohne Erfolg mit Empl. de Goa.

Havas zerstörte die Knoten mit 10\% iger Pyrogallussalbe.

Zur Linderung der subjektiven Beschwerden wandte Kutiak (50) warme Bäder mit gutem Erfolg an.

Schwimmer (51) empfahl auf Grund guter eigener Erfolge das Phenacetin zur Linderung der Schmerzen. 
Gute Erfolge sahen wir von der Röntgenbestrahlung. Dieselbe wurde in der Weise ausgeführt, daß bestimmte Bezirke der Wirkung der X-Strahlen für die Dauer von 10-15 Min. ausgesetzt wurden. Die angewandte Stromstärke betrug 3 Milli-Ampère, der durchschnittliche Abstand $10-20 \mathrm{~cm}$. Die Reaktion wurde nach einmaliger Bestrahlung abgewartet und alsbald zeigte sich jedesmal, daß in dem jeweiligen, durch seine Rötung deutlich gegen die in der üblichen Weise abgedeckten Hautpartien sich abhebenden Bezirke die Tumoren allmählich kleiner wurden, während die in der Nachbarschaft befindlichen nicht bestrahlten Knoten unbeeinflußt blieben.

Mit der Rückbildung der bestrahlten Tumoren bzw. Infiltrate trat gleichzeitig eine Depigmentation in der Weise ein, daß die zentral gelegenen Partien zuerst abblaßten und die ursprünglich diffuse dunkle Verfärbung sich schließlich auf die Ränder beschränkte. Auf diese Weise entstanden hellbraune Pigmentflecke, die von einem dunkelbraunen Saum begrenzt wurden. Die Infiltrate waren an diesen Stellen, die außer geringer Atrophie und der geschilderten Pigmentierung nichts Abnormes mehr boten, schließlich völlig verschwunden.

Wenn wir auf Grund dieser guten Erfahrungen die Röntgentherapie empfehlen, so sind wir uns doch der Grenzen des damit zu Leistenden wohl bewußt:

Der durch das Auftreten von Tumoren in inneren Organen beringte ungünstige Ausgang des Leidens wird auch durch dieses Mittel nicht verzögert werden, doch können wir durch dasselbe die subjektiven Beschwerden vielfach lindern und durch Ermutigung der über den sichtlichen Erfolg stets erfreuten Kranken viel zur Erhaltung ihres Wohlbefindens beitragen.

In vielen Fällen werden auch Dunstumschläge, Salbenverbände, die Darreichung von Analgeticis und eine gute Ernährung von Nutzen sein. fehlen.

Die Anwendung des Arsens ist in jedem Falle zu emp-

Hier und da dürfte auch die Exzision des einen oder anderen schmerzhaften Knotens ratsam sein. So haben z. B. wir wiederholt die bei unseren Patienten durch ihre Lokalisa- 
tion besonders schmerzhaften, an der Fußsohle sitzenden Knoten exzidiert, was freilich meistens mit kolossalen Blutungen verbunden war, aber doch stets große Erleichterung brachte.

Nach dieser Übersicht über die den Kliniker interessierenden Einzelheiten wenden wir uns den histologischen Befunden zu. Dieselben sind innerhalb dreier Jahrzehnte immer zahlreicher und auch immer umfangreicher geworden, so daß mit der ausführlichen Wiedergabe aller sich Bände füllen ließen.

Im Interesse einer möglichst übersıchtlichen und klaren Darstellung mïssen wir uns darauf beschränken, stets nur die wichtigsten Punkte hervorzuheben. Um dieser Aufgabe genügen zu können, bemerken wir schon jetzt, daß den Kernpunkt aller mehr oder weniger umfangreichen histologischen Beschreibungen heutigen Tages lediglich die Frage darstellt:

Handelt es sich bei den Tumoren des Typus Kaposi um echte Sarkome oder um entzündliche Produkte (Granulationsgeschwülste)?

Indem wir von diesem Gesichtspunkte aus eine Scheidung der Anhänger dieser beiden Haupttheorien vornehmen, beginnen wir wiederum mit der Ansicht $K$ a p o sis.

Dieser Autor schreibt:

„Histologisch stellt sich dasselbe (d. Sarkom) durchwegs dar als Rundzellensarkom, doch findet sich stellenweise auch charakteristisches Spindelzellensarkom. Eigentümlich diesem Typus sind mikroskopisch erweislich interstitielle Kapillarhämorrhagien, woher die spätere schwarzblaue Pigmentierung der anfangs mehr lebhaften Knoten erklärlich erscheint."

$\mathrm{Babès} \mathrm{(52)} \mathrm{und} \mathrm{Bernhardt} \mathrm{(53)} \mathrm{nennen} \mathrm{nach} \mathrm{ihren} \mathrm{bisto-}$ logischen Untersuchungen die Kaposi sche Krankheit „Angiosarcoma periteliale fusocellulare".

R i ehl (54) ist der Ausicht, daß die Tumoren als Angiome beginnen, die aber sofort das Charakteristische der malignen Neubildung an sich tragen, indem neben den Gefäßanlagen sofort die Sarkomzellen auftreten.

Auch Joseph (55) hält die Tumoren für Angiosarkome.

Rundzellensarkome fanden u. a. Ka p o si, Perrin, Jordan (56), Un na.

Ferner fanden Sarkome und zwar meistens Spindelzellensarkome: Metscherski, Philippson, Bernhardt, Johnston und $\mathrm{K}$ öhler (57), D e A m i c is, F unk (58), U i or th, Havas, Köbner, Lustgarten (59), S chwimmeru. a. 
Pelagatti zählt, ebenfalls von seinen 7 Fällen von Hautsarkomatose 3, welche dem uns ausschließlich beschäftigenden Typus $\mathrm{K}$ a posi entsprechen, zu den Sarkomen; sein vierter, auch klinisch vom Typus Kaposi abweichender Fall stellt ein Riesenzellensarkom dar. Die Tumoren seiner 3 übrigen Fälle sind nach seiner Ansicht Granulationsgeschwülste.

Spiegler (60) sieht seine als IV, V, VI publizierten Fälle von Sarcomatosis cutis, welche klinisch durchaus dem $K$ a $p$ os i schen Typus entsprechen, nicht als Sarkome an, sondern erklärt die Tumoren als „ganz streng circumscripte, nicht fortschreitende, wohl aber der Rückbildung fähige Bindegewebswucherungen mit kleinzelligem Infiltrate", welche aber seiner Ansicht nach "Übergangsformen von entzündlicher Wucherung (chronischer Entzündung) zu den Sarkomen darstellen mögen".

Im Falle von $\mathrm{Ku}$ dis ch (61) wies das mikroskopische Bild ebenfalls auf einen chronisch entzündlichen Granulationsprozeß hin.

Sequeir und Bulloch fanden bei der Untersuchung ihres Falles ein Gewebe von eher chronisch eutzündlichem als sarkomatösem Charakter.

Gottheil (62) fand gruppenweise Anhäufungen von meistens rundlichen Bindegewebszellen, die aber nicht das Bild eines echten Sarkoms boten.

J a d a s o b n (63) hält es für eine noch der Entscheidung harrende Frage, ob es sich bei der $\mathrm{K}$ ap o s i schen Krankheitsform um Angiosarkome oder entzündungsartige resp. infektiöse Neubildungen handelt.

Sellei fand in seinen Fällen, welche klinisch durchaus der Kap o s i schen Beschreibung entsprachen, mikroskopisch niemais Anhalispunkte dafür, daß es sich um echte Sarkome handle, und teilt auf Grund seiner Untersuchungsresultate die Ansicht $\mathrm{T}$ ör öks (64), K ö b n er s, Steiners (65), Petersens u. a, daß die Kaposische Krankheit nicht zu den Sarkomen gehöre.

Auch Kundrat, Paltauf und Majoc cbi sondern sämtliche Hautsarkome von der Sarkomgruppe $a b$ und zählen sie zu den Granulomen.

Bei der vorstehenden Übersicht über die histologischen Befunde der einzelnen Autoren haben wir, um die bestehende Verwirrung nicht noch zu erhöben, jene Fälle unberücksichtigt gelassen, deren klinische Beschreibung sich nicht völlig mit den von Kapos i aufgestellten Angaben über das idiopath. multiple Pigmentsarkom deckt, oder welche von den Autoren selbst als abweichende Formen bezeichnet werden, z. B. die Fälle von Fendt (66), Tandler (67) und Krzystal l o w i ez (68).

Ob der von Cholin (69) vorgestellte Fall, der von ihm selbst als in die bisher beschriebenen Sarkomgruppen nicht passend angesehen, von $\mathrm{Tschle} n$ off und $\mathrm{P}$ ospelow aber für Sarkom gehalten wurde, zum Typus Kapos j gehört oder nicht, können wir nicht entscheiden. 
Unsere eigenen histologischen Untersuchungen nahmen wir an Tumoren vor, die aus therapeutischen Gründen excidiert worden waren und fast ausschließlich an der Fußsohle saßen. In der Entstehung begriffene Knötchen zu erbalten, war leider in keinem unserer Fälle möglich, jedoch bot die Untersuchung auch der ausgebildeten Knoten nebst ihrer nächsten Umgebung so charakteristische Befunde, daß wir durch eine Schilderung derselben zur Histologie des Sarcoma idiop. multipl. haem. beitragen zu können glauben.

Zur Besprechung wählen wir zunächst ein Präparat, welches sich schon bei äußerer Besichtigung als Durchschnitt durch einen Tumor erweist, der durch seine Erhebung über das Hautniveau und eine stärkere Hornschicht von seiner Umgebung abgegrenzt erscheint.

Schon bei schwacher Vergrößerung fällt zunächst in der Nachbarschaft des Knotens eine große Anzahl mit roten Blutkörperchen gefüliter Räume ins Auge, welche dieganze Cutıs durchsetzen und bis in die Papillarschicht reichen. Die Papillen sind vielfach verstrichen und die Grenze zwischen Cutis und Epidermis entbehrt der charakteristischen Zeichnung. Die Epidermis selbst erscheint im ganzen verschmälert; nur die Hornschicht ist verdickt.

Die von den erwähnten Bluträumen nicht eingenommenen Teile der Cutis erweisen sich überaus zellreich.

Bei starker Vergrößerung kann man erkennen, daß die erwähnten bluthaltigen Räume teils aus abnorm erweiterten, teils aus neugebildeten Gefäßen bestehen. Das Endothel ist meistens gut erhalten, an manchen Stellen zeigen sich jedoch im Gefäßrohr deutliche Lücken, durch welche das Blut sich in das umgebende Gewebe ergossen hat. Zuweilen kommunizieren auch die größeren Bluträume durch derartige Lücken miteinander.

Eine genauere Betrachtung der, wie erwähnt, zellreichen Umgebung, der teils dilatierten, teils neugebildeten Kapillaren ergibt, daß die meist spindelförmigen Zellkerne in Zügen angeordnet sind, die vielfach dem Verlauf der Kapillaren entsprechen; an anderen Stellen wiederum erscheinen sie mehr regellos oder im Querschnitt getroffen. Zwischen diesen Zellen findet sich an vielen Stellen ein zartes, zuweilen auch gröberes Fibrinnetz, dessen Maschen wiederum häufig mit zahllosen roten Blutkörperchen und vereinzelten Lymphocyten gefïllt erscheinen. In den tieferen Cutisschichten kommen die Gefäßveränderungen weniger als Dilatation oder Neubildung, sondern vielmehr als kolossale Wucherung des Perithels und Verdickung des Gefäbrohrs durch Quellung der Endothelien, vor allem aber durch Wucherung der Media zum Ausdruck. 
Die geschilderten, in langen sich vielfach verflechtenden Zügen angeordneten, Zellwucherungen finden sich am reichlichsten in dem deutlich gegen seine Umgebung abgesetzten Knoten selbst wieder. Sie bilden sozusagen die Grundsubstanz des Tumors. Innerhalb desselben weisen die Zwischenräume der Zellen - im Gegensatz zu der oben geschilderten Umgebung des Tumors - nur selten frische Blutungen in Gestalt wohlerhaltener Blntkörperchen auf; hier erscheinen die Zwischenräume vielmehr an vielen Stellen durch ein weit gröberes vielmaschiges Fibrinnetz ausgegossen.

Die auch oft zahlreich innerhalb des Tumors zu findenden, oben beschriebenen - aber hier viel kleineren - Räume enthalten dagegen meist noch gut erhaltene Erythrocyten.

Von besonderem Interesse ist noch das Verhalten der elastischen Fasern: Innerhalb des Tumors fehlen dieselben völlig. Dagegen verhalten sie sich in der Umgebung der Knoten normal, abgeseben von den Stellen, an denen sich die erwähnten Wucherungen der Gefäßperithelien vorfinden.

Mast- und Plasmazellen fanden sich nie in auffallender Zahl.

In der Umgebung der erwähnten durch Kapillarzerreißung entstandenen Blutungen ins Gewebe war häufig Pigment in größerer Menge nachwcisbar.

Bei der obigen Skizzierung des histologischen Befundes haben wir absichtlich die Veränderungen innerhalb des Tumors denen seiner Umgebung zum Vergleich an die Seite gestellt, da letztere u. E. jene Vorgänge erkennen läßt, durch die es schließlich zur Bildung eines derben Knotens kommt.

Betrachten wir zunächst die unmittelbare Umgebung des Tumors, so ist der Reichtum an neugebildeten Gefäßen so auffallend, daß sofort der Gedanke an ein Angiom auftaucht. Außer der Gefäßvermehrung sehen wir aber auch an vielen Stellen eine deutliche Wucherung der Perithelien, die durch exzessive Zellvermehrung schließlich zur Bildung derberer Tumoren führt. Wir haben es also mit einem Angiosarkom zu tun. Um diese Diagnose zu begründen, müssen wir etwas weiter ausholen.

Bei genauem Studium der wichtigsten, in letzter Zeit erschienenen Publikationen fiel uns auf, daß die ausführlichere Schilderung der histologischen Befunde in den meisten Fällen auf gleichartige oder mindestens sehr ähnliche mikroskopische Bilder zurückzuführen, daß nur ihre $\mathrm{D}$ e utu ng eine verschiedenartige war: wäbrend ein Teil der Autoren sich für die Diagnose "Sarkom" entschied, erklärten andere für Sarkom nicht den geringsten Anhaltspunkt gefunden zu haben. Wie ist das zu erklären? 
Man fand in der Hauptsache neugebildete und erweiterte Gefäße und in der Umgebung derselben viele Zellen: „Entzündung" sagten die einen, ,Sarkom" die anderen.

Da nicht anzunehmen ist, daß die Produkte einer klinisch so gut abgegrenzten Krankheit sich bald als Sarkome, bald als entzündliches Granulationsgewebe manifestieren, so bleibt uns nichts anderes übrig als die große Ähnlichkeit vieler entzündlicher Prozesse mit manchen echten Neubildungen - soweit sie bindegewebiger Natur sind - zur Erklärung dieser Meinungsverschiedenheiten heranzuzieben. Wir zitieren in folgendem eine Äußerung Kolas ceks (70), die sich hierauf bezieht:

"Was den Angiosarkomen in meinen Augen noch einen besonderen Wert verleiht, das ist das an die Entzündung mahnende mikroskopische Bild ihrer Wachstumanfänge. Die mikroskopischen Kardinalsymptome einer Entzündung, nämlich Erweiterung und pralle Füllung der Gefäße, hauptsächlich aber die mantelförmige Anbäufung von Zellen um dieselben, hat die in Rede stehende Geschwulstbildung (das Angiosarkom) mit der Entzündung gemein.

Manchmal sind noch die Geschwulstzellen allem Anscbein nach mit Granulationszellen identisch, so daß die Ähnlichkeit mit den Entzündungsprodukten noch gesteigert wird."

Nun haben wir es aber in unserem Falle nicht nur mit einer "Erweiterung und prallen Füllung der Blutgefäße", sondern mit einer dentlichen Vermehrung resp. Neubildung derselben zu tun, da der große Reichtum an Gefäßquerschnitten, die wir in unseren Präparaten fanden, keineswegs lediglich auf Erweiterung autochthoner Kapillaren, sondern vielmehr auf wirkliche Vermehrung derselben zurückzuführen war und zwar in so hohem Grade, wie es niemals bei Entzündung, sondern nur bei echten Gefäßgeschwülsten, bei Angiomen, vorkommt.

Betrachten wir jetzt die in der Umgebung jener Gefäßse angebäuften Zellen, so fällt allerdings auf den ersten Blick eine gewisse Ähnlichkeit mit solchen Bildern ins Auge, wie sie uns von der mikroskopischen Untersuchung entzündlicher Vorgänge her gelänfig sind. Bald aber erkennen wir, da $B$ die in nächster Umgebung der Gefäße angehäuften Zellen mit rundem Kern keineswegs alle den bei entzündlichen Vorgängen sich findenden per diapedesin ausgewanderten weißen Blutzellen entsprechen, sondern zum größten Teil junge Bindegewebszellen sind, die in ihrer mehr rundlichen Gestalt, wie man sie in embryonalem Gewebe findet, das jüngste Stadium ihrer Entwicklung darstellen. Sie liegen ja noch in nächster Nähe ihrer Mutterzellen, nämlich jener Zellen, aus denen sich dic Kapillaren zusammensetzen, die in ihrer einfachsten Gestalt 
gewissermaßen einen Protoplasmaschlauch mit eingestreuten Zellkernen darstellen. Daß die fraglichen Zellen tatsächlich von der Wand der Blutgefäße aus gewucherte junge Bindegewebszellen sind, scheint uns bewiesen durch ihre dem Verlauf der GefäBe entsprechende Anordnung und angesichts der Tatsache, daß die jüngsten, gleichsam embryonalen, noch nicht differenzierten Zellen mit rundem Kern den Gefäßen am nächsten liegen, während die spindelförmigen, in dichten Zügen angeordneten, die älteren Zellindividuen darstellen.

Wir haben es also mit exquisiter Neubildung von GefäBen und von Bindegewebselementen zu tun und nicht mit einer entzündlichen Infiltration des autochthonen Gewebes.

Unsere Abbildung (s. Tafel) zeigt nun in anschaulicher Weise die oben geschilderte Erweiterung und Vermehrung der Blutgefäße, sowie in deren Umgebung jene Zellwucherungen, die in der Tiefe in einem größeren Bereich bereits eine solche Dichtigkeit erlangt haben, wie wir sie in den bereits länger bestehenden derben Knoten durchweg anzutreffen pflegen. Der Schnitt stammt von einem relativ jungen Knoten, d. h. von einem solchen, der sich zwar bereits deutlich über das Hautniveau erhob und im Gegensatz zu den in Entstehung begriftenen hellroten Tumoren bereits eine blaue Färbung zeigte, im übrigen aber noch jene charakteristische schwammartige Konsistenz aufwies wie wir sie im klinischen Teil beschrieben haben. Weitere Einzelnheiten der Abbildung erklären sıch an der Hand unserer histologischen Schilderung von selbst.

\section{Resumé.}

Aus dem Resultat unserer klinischen Beobachtungen und mikroskopischen Untersuchungen möchten wir noch folgende Punkte hervorheben:

Wir halten das Sarcoma id. m. h. für eine exquisite Erkrankung des Gefäßsystems, indem wir das letztere trotz der üblichen anatomisch und physiologisch gerechtfertigten Trennung in Arterien, Venen, Kapillaren und Lymphgefäße als eine Einheit ansehen, dessen einzelne nur durch Differenzierung von einander abweichenden Abschnitte bald in ihrer Gesamtheit, bald einzeln die geschilderten pathologischen Vorgänge aufweisen können. Wie erwähnt, beobachteten z. B. Philipps on und Bernh ard t eine ganz auffallende Alteration der Lymphgefäße; in anderen Fällen wiederum trat eine solche mehr in den Hintergrund. 
Nehmen wir somit das ganze Gefäßsystem als Ausgangspunkt der pathologischen Produkte an, so tritt uns zuerst die Frage entgegen: Durch welche Noxe werden diese Vorgänge ausgelöst?

Die Ansicht so vieler Autoren, daß es sich dabei um die Tätigkeit von Mikroorganismen handle, vermögen wir nicht zu teilen. Selbst aber wenn es sich in unserem Falle wirklich um eine Infektionskrankheit handeln sollte, so reicht doch die Annahme einer spezifischen Art von Bakterien allein zur Erklärung der Krankheitserscheinungen nicht aus, denn gerade bei Infektionskrankheiten stehen sich zwei Faktoren gleichwertig gegenüber: Die Disposition und die veranlassende Schädlichkeit.

Was nun bei den an Sarcoma id. m. h. leidenden Kranken zunächst die Disposition betrifft, so könnte dieselbe z. B. angeboren sein, oder - wie oben erwähnt -- in der Rasse ihre Erklärung finden, vielleicht auch in der Ernährung, wie sie durch Klima, Bodenbeschaffenheit und Lebensgewohnheit bedingt wird. Wir erinnern daran, daß diese Krankheit vorzugsweise im Osten Europas und vielfach bei Juden vorkommt.

Wir glauben, daß gerade bezüglich der Disposition sich noch wertrolle Aufschlüsse gewinnen lassen werden, sobald man sich wieder mehr daran gewöhnt haben wird, die Ursachen mancher pathologischen Erscheinungen mehr aus den komplizierten Vorgängen im Organismus zu erklären, anstatt sie allein in einer Invasion von Parasiten zu suchen.

Bezüglich der veranlassenden Schädlichkeit erinnern wir daran, daß in manchen Fällen wiederholte Durchnässungen resp. Erkältungen der Füße den ersten Krankheitserscheinungen voraufgegangen waren. Daß derartige Beobachtungen sicherlich keine Zufälligkeiten darstellen, darauf weist z. B. die Angabe eines unserer Patienten hin, daß jedesmal nach einem kalten Bade sein Zustand durch Zunahme der Knoten sich erheblich verschlimmert habe. Freilich reicht der in solchen Fällen anzunehmende Reiz, den Temperatureinflüsse auf ein labiles Gefäßsystem ausübten, keineswegs für alle derartigen Erkrankungen aus.

In einem Falle Bernhardts zeigten sich die ersten 
Sarcoma idiopathicum multiplex haemorrhagicum (Kaposi). 401

Symptome an einer Stelle, wo erst kurz vorher ein Erysipel abgelaufen war. Auch in diesem Falle wird es uns weit leichter, die durch ein Erysipel bedingte Alteration der Gefäße als veranlassende Krankheitsursache anzusehen, als uns die Tätigkeit unbekannter Mikroorganismen vorzustellen, für deren Existenz bisher noch nicht die geringsten Anhaltspunkte erbracht worden sind.

Wir sind also der Ansicht, daß die Ursachen, welche das Leiden hervorrufen, mannigfacher Natur sein können und daß bei in gleicher Weise disponierten Individuen bald das eine, bald das andere auslösende Moment in Wirksamkeit treten und die ersten Krankheitserscheinungen hervorrufen kann.

Ferner glauben wir, daß bei der uns beschäftigenden Krankheit die Tumorenbildung keineswegs immer die Antwort auf einen von außen kommenden Reiz zu sein braucht, sondern oft auf Vorgänge im Organismus selbst zurückzuführen sein wird. Wir erinnern in dieser Beziehung an die glänzende Abhandlung Paltaufs ,über die lymphatischen Neubildungen der Haut", in welcher er darauf hinweist, daß $25 \%$ der an Leukaemie Erkrankten schwere Intermittens durchgemacht hatten und daß die Ansprüche, welche zur Regeneration an die blutbereitenden Organe gestellt wurden, bei gewissen Individuen wahrscheinlich den Reiz zu übermäßiger Bildung abgaben, oder - mit anderen Worten - daß gewisse Regenerationsvorgänge über die Norm hinausschießen können. Da nun auch wir beim Studium der Literatur und in unseren Krankengeschichten nicht selten die anamnestische Angabe fanden, daß die Patienten an schweren Krankheiten (Malarja, Cholera) gelitten hatten, so halten wir es für möglich, daß auch in diesen Fällen durch das Bestreben des Organismus die Blutbeschaffenheit wieder auf ihre frühere Höhe zu bringen, der erste Anstoß zu Wucherungen im Gefäßsystem bei dazu besonders disponierten Individuen gegeben wurde.

Indem wir uns jetzt den ersten Anfängen der Krankheit selbst zuwenden, heben wir nochmals hervor, daß die ersten Geschwulstanlagen Wucherungsvorgänge an den Gefäßen mit Neubildung solcher darstellen, so daß die histologischen Bilder den Eindruck von Angiomen hervorrufen, die aber sehr bald 
durch Wucherung der Perithelien den Charakter der Angiosarkome annebmen. Aus dem kolossalen Reichtum an neugebildeten Gefäßen ist die anfänglich teigige Beschaffenheit der Tumoren sowie ihre blaurote Farbe zu erklären, während die bäufig schmutzig grünliche Verfärbung ihrer Umgebung auf Blutungen in das Gewebe zurückzuführen ist, wie wir sie in unseren Präparaten häufig beobachteten.

Mit zunehmender Zellwucherung innerbalb der Knoten nimmt auch ihre Konsistenz $\mathrm{zu}$, die sie längere Zeit beizubehalten pflegen. Die häufig erst nach Monaten erfolgende Involution der Tumoren ist einerseits sicherlich die Folge regressiver Vorgänge, wie sie auch sonst bei Sarkomen und Carcinomen allgemein bekannt sind, andrerseits erklären wir uns dieselbe dadurch, daß durch Zerreißung der prall gefüllten Kapillaren häufig Blutungen ins Gewebe stattfinden, wodurch die betreffenden Gefäße zu Grunde gehen und gleichzeitig die vorher von ihnen ausgegangene Zellwucherung zum Stillstand gelangt. Wir sehen in der Tatsache, daß die Knoten nicht immer ins Grenzenlose wachsen und ohne Ulzeration wieder scheinbar völlig verschwinden können, keinen Grund, dieselben avs der Reihe der echten Geschwülste überhaupt zu streichen.

Wenn von vielen Autoren darauf hingewiesen wird, daß schon Virchow betonte, man müsse zur Charakterisierung mancher Geschwülste auch ihr klinisches Verhalten beranziehen und demzufolge die Tumoren dieses Leidens wegen ihrer Fähigkeit sich fast völlig zu involvieren, nicht zu den echten Geschwülsten zählen wollen, so möchten wir demgegenüber folgendes hervorheben:

Es ist gewiß richtig, daß in vielen Fällen die Tumoren des Sarcoma id. m. h. sich hochgradig involvieren, ja daß sie sogar scheinbar völlig verschwinden können. U. E. beruht aber diese Erscheinung $u$. a. darauf, da $\beta$ die im Zentrum der Knoten zuerst sich bildenden Produkte der regressiven Metamorphose sowie die durch die erwähnten Blutungen zerstörten Gewebselemente außergewöhnlich leicht zur Resorption gelangen können wegen des kolossalen Reichtums der Tumoren und ihrer Umgebung an Lymph- und Blutwegen. Ebenso scheint uns in dem Umstande, daß auch die Peripherie und nächste Umgebung 
Sarcoma idiopathicum multiplex haemorrhagicum (Kaposi). 403

der Knoten so überaus reich an Blutgefäßen zu sein pflegt und daher unter relativ guten Ernährungsverhältnissen sich befindet, auch die Tatsache hinreichend erklärt, daß die Tumoren so selten spontan ulzerieren.

Im Gegensatz zu der in der Literatur häufig hervorgehobenen spontanen Involution der Tumoren möchten wir aber doch hervorheben, daß manche Knoten zwar mit Bildung einer zentralen Delle sich zum Teil zurückbilden, dafür aber nach der Tiefe zu ein unbegrenztes Wachstum zeigen können. Wir erinnern in dieser Hinsicht an die völlige Zerstörung von Knochen in einem Falle Bernhardts und bei einem unserer Patienten.

Gerade die Tatsache, daß die Zellwucherungen sich nicht immer auf die Haut beschränken, sondern auch die tiefer liegenden Organe durchsetzend Múskeln und Knochen zerstören können, beweist, daß wir es mit echten malignen Neubildungen zu tun haben. Sowohl die Resultate der anatomischen Untersuchungen als auch die klinischen Beobachtungen führen uns zu dem Schlusse, daß wir diese eigentümliche Krankheit als eine besondere Form der "Sarcomatosis cutis" aufzufassen haben, wie dies Kaposi bei seiner ersten Schilderung bereits getan hat. Inwieweit andere Formen der "Sarcomatosis cutis" mit dem Typus Kaposi verwandt sind oder Übergänge $z \mathfrak{u}$ demselben darstellen, wird vielleicht durch spätere Untersuchungen festgestellt werden können. Nach dem Stande unserer heutigen Kenntnisse sind wir durchaus berechtigt, für das Sarcoma idiopathicum multiplex haemorrhagicum eine Sonderstellung in Anspruch zu nehmen.

Am Schlusse meiner Arbeit angelangt erlaube ich mir Herrn Prof. Dr. Riehl für die gütige Überlassung des Materials, wie auch für die vielfache Anregung und mir gewidmete Zeit meinen verbindlichsten Dank auszusprechen. 


\section{Literatur.}

1. Kaposi. Idiopath. multiples Pigmentsarkorn der Haut. Archiv für Dermat. und Syph. 1872.

2. Kaposi. Pathologie und Therapie der Hautkrankheiten.

3. Unna. Die Histopathologie der Hautkrankheiten.

4. Perrin, De la dermatose cutanée. Paris 1886.

5. Schwimmer. Archiv für Dermat. und Syph. Bd. XXXVII. 1896. pag. 245.

6. De Amicis. Sulla sarcomatosi cutanea idiopatica. - Gazzetta degli ospedali e delle cliniche. Napoli, 1897. Nr. 64. pag. 685.

7. Pelagatti. Die Hautsarkomatose. Monatshefte f. prakt. Dermat. Bd, XXXV. 6. Heft, pag. 497.

8. Li i berthal. Sarcomatosis cutis. Amer. Med. Assoc. Journ. 1902. Nr. 28.

9. Sellei. Über das idiopath. Kaposische Sarcoma multiplex haemorrhagicum. Monatsh. f. prakt. Dermat. 1900. Bd. XXXI. pag. 413.

10. Pospelow. Vener.-dermat. Gesellschaft zu Moskau. 8. April 1898.

11. Pringle. Congrès international de dermatologie. Paris 1889

12. Philippson. Über das Sarcoma idiopathicum cutis Kaposi. Ein Beitrag zur Sarkomlehre. Virchows Archiv. Bd. CLXVII. pag. 58.

13. Semenow. Zur Pathologie des Sarcoma pigmentoides idiopathicum multiplex cutis Kaposi. Tagebuch des VI. Pirogoffschen Kongresses. Kiew 1896.

14. Campana. Über Sarkomatose der Haut. XII. Kongreß der ital. Ärzte zu Pavia 1897.

15. Tinzew. Aus der Gesellschaft für wissenschaftl. Medizin und Hygiene a. d. Universität Charkow, Oktober 1896.

16. Paltauf. Über lymphatische Neubildungen der Haut. II. int. Dermatologenkongreß. Wien 1892.

17. Köbner. Verhandlungen der dermat. Sektion beim $X$. internationalen Kongreß zu Berlin 1890.

18. Se m e now. Zehn Fälle des Sarcoma idiop. pigm. multipl. cutis. Monatsh. f. prakt. Dermat. 1897. Bd. XXV. pag. 539.

19. Sellei. Weitere Beiträge zur Patbologie des sog. Sarcoma multiplex pigment. haemorr. idiop. (Kaposi). Archiv f. Dermat. und Syph. 1903. Band LXVI. pag. 41.

20. Kaposi. Atti dell XI Congresso Medico internationale, Roma 1895.

21. Schwimmer. Über das Pigment-Sarkom und einige verwandte Affektionen. Third international Congress of Dermatology. London 1896.

22. Bernhardt. Weitere Mitteilungen über Sarcoma idiopathicum multipl. pigmentos. cutis. Arch. f. Derm. u. Syph: 1902. Bd. LXII. pag. 237. 
Sarcoma idiopathicum multiplex haemorrhagicum (Kaposi). 405

23. Ka lin dero. Sarcome cutané pigmentaire multiple idiopathique, avec début par les extrémités (Type de Kaposi) Congrès intern. de dermat. Paris 1889. pag. 752 .

24. Sedziak. Seltener Fall von Sareomata multiplicia cutis et lymphosarcoma tonsillae extrae. Gazeta Lekarska Nr. 44. 1892.

25. Scholtz. Verhandlungen der Breslauer dermat. Vereinigung. Januar 1900.

26. Géza Dieballa. Über einen mit Lymphocytose einhergehenden Fall von Sarcoma multiplex cutis. Wiener kl. Wochenschrift 1897. pag. 22.

27. Tanturri. Über das idiopath. kleinzellige, teleangiektatische Pigmentsarkom der Haut. Il Morgagni. Juliheft 1877.

28. Sellei. Beiträge zur Pathologie des Kaposischen „Granuloms." Monatsh. f. prakt. Derm. 1902. Bd. XXXIV. pag 497.

29. Philippson. Ein Fall von Sarkomatose der Haut verbunden mit visceraler Carcinomatose. Rif. med. 1898. Nr. 165.

30. Bernhardt. Sarcoma idiopathicum multiplex en plaques pigmentosum et lymphangiectodes. Eine eigentümliche Form der sogen. Sarcomatosis cutis. Archiv für Derm. u. Syph. 1902. Bd. LXIII. pag. 239. 31. Sedziak. ef. 24.

32. Kaposi. Verhandlungen der Wiener dermatolog. Gesellschaft. 26. April 1899.

33. Stanziale. Klinischer Bericht über 410 dermo-syphilidol. Krankheitsfälle. Gazz. osped. 1890.

34. Langenbuch. Heilung recidivierter multipler Sarkome der Hautdecken nach einem Erysipelas migrans.

35. Coley. Behandlung von Sarkomen mit den Toxinen des Erysipels und des Bacillus prodigiosus. Amer. med.-surg. bull. 1895, Nr. 9. 36. J a cks on. Verhandlungen der New-York Dermatological Society, 26. Jan. 1897.

37. Elli ot. Ibidem.

38. Havas. Ungar. dermat. u. urolog. Gesellschaft in Budapest. April 1899.

39. D'Antona. Sul sarcomatosi cutanea multipla. Gazzetta degli ospedali e delle cliniche Napoli 1897.

40. Sequeir u. Bulloch. Ein Fall von idiop. multipl. Pigmentsarkom. The British Journal of Dermatologg 1901 (Juni). Bd. XIII.

41. Köbner. Heilung eines Falles von allgem. Sarkomatose der Haut d. subkutane Arseninjektionen. Berliner klin. Wochenschr. 1883, Nr. 2.

42. Havas. Prager med. Wochenschrift 1892, Nr. 48.

43. Viganò. Ein Fall von Sarcoma idiopathicum cutis. Il Morgagni 1903, Nr. 1.

44. Metscherski. Venerol.-dermatolog. Gesellschaft zu Moskau. Februar 1900.

45. Trapesnikow. Prot. d. St. Petersb. russ, syph. und derm. Ges. 1895/96. Band XI. 
46. Pospelow. Zur Kasuistik der mit Arsen behandelten primären Hautsarkome. Festschrift Neumann 1900.

47. Kracht. Prot. der Moskauer derm. u. vener. Ges. 1896/97 u. April 1898.

48. Sherwell. Verhandl. d. New-York Dermatological Society Januar 1897.

49. Uiorth. Et Tilsolde of idiopatik multipell Pigmentsarkom (Kaposi) Klinik Aarbog. Christiania 1886.

50. Katiak. Wiener med. Wochenschrift 1893, Nr. 40.

51. S chwi m mer. Sarcoma pigm. diffusum multipl. Internationaler Atlas selt. Hautkrankheiten. Hamburg 1890.

52. B abè s. Zentralblatt für die med. Wissenschaft. 1883.

53. Bernhardt. Sarcomata idiop. multiplicia pigm. cutis (Kaposi).

Archiv f. Derm. u. Syph. 1899. Bd. XIIX. pag. 207.

54. Riehl. Verhandlungen der Wiener dermatolog. Gesellschaft, 23. März 1892.

55. Jo s e ph. Lehrbuch der Hautkrankheiten.

56. Jordan. Vener.-derm. Ges. zu Moskau 1901.

57. Johnston u. Köhler. Journal of cutan. and genito-urinary diseases. Jänner 1902.

58. F u nk, Klinische Studien über Sarkome der Haut. Monatsh. f. prakt. Dermat. 1889.

59. Lustgarten. Verhandl. der New-York Dermat. Society. November 1897.

60. Spiegler. Über die sogen. Sarcomatosis cutis. Archiv f. Derm. v. Syph. 1894. Bd. XXVII. pag. 163.

61. Kudisch. Dermat. Zeitschrift 1902. 3. Heft. pag. 402.

62. Gottheil. Journal of cutan. and genito-urin. diseases 1902.

63. Jadassohn. Handbuch der prakt. Medizin. (SchwalbeEpstein) 1901.

64. Török. Zwei F'älle von Sarcoma idiopathicum multiplex. Festschrift Neumann.

65. Steiner. Berliner kl. Wochenschr. 1896. Nr. 28.

66. F e ndt. Beiträge zur Kenntnis der sogenannten sarkoiden Geschwülste der Haut. Archiv f. Derm. und Syph. 1900. Bd. LIII. pag. 213.

67. T a ndler. Beitrag zur Kenntnis der Sarcomatosis cutis. Archiv f. Derm. u. Syph. 1897. Bd. XLI. pag. 163.

68. Krzystallowicz. Przeglad lekarski 1903. Nr. 49-51.

69. Cholin. Vener. dermatol. Gesellschaft zu Moskau. März 1900.

70. Kolaczek. Über das Angiosarkom. Deutsche Zeitschrift für Chirurgie. IX und XIII.

Die Erklärung der Abbildung auf Taf. $X$ ist dem Texte zu entnehmen. 


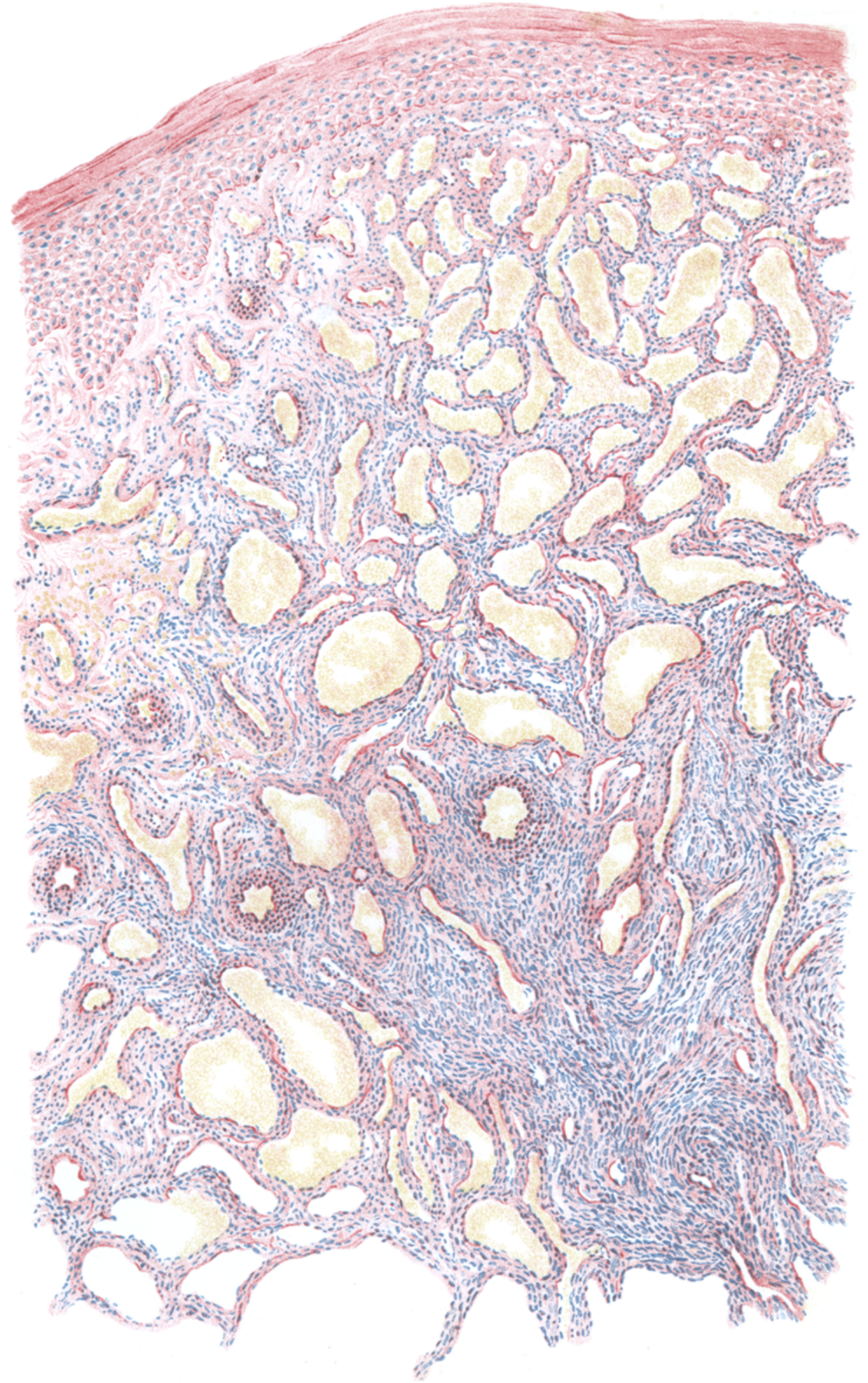

